\title{
Suplementação oral de magnésio na prevenção de cãibras musculares: revisão baseada na evidência
}

Cátia Palha, ${ }^{1}$ Miguel Gouveia, ${ }^{2}$ Sara Guimarães Fernandes ${ }^{2}$

\section{RESUMO}

Objetivo: Rever a evidência sobre a eficácia da suplementação oral de magnésio na prevenção de cãibras musculares, sem patologia evidente associada, em indivíduos adultos.

Fontes de dados: National Guideline Clearinghouse, Canadian Medical Association Practice Guidelines Infobase, Guidelines Finder, The Cochrane Library, DARE, Bandolier, Evidence-Based Medicine Online e PubMed.

Métodos: Pesquisa realizada no dia 4 de julho de 2018 com os termos MeSH muscle cramp, magnesium e magnesium compounds de meta-análises, revisões sistemáticas, ensaios clínicos aleatorizados e controlados (ECAC), estudos de coorte e caso-controlo e normas de orientação clínica publicados desde 1 de janeiro de 2008 nas línguas portuguesa, inglesa e espanhola. Para atribuir níveis de evidência (NE) e forças de recomendação foi utilizada a Strength of Recommendation Taxonomy (SORT), da American Academy of Family Physicians.

Resultados: Dos 47 artigos encontrados, cinco cumpriram os critérios de inclusão: uma meta-análise (NE 2), três revisões sistemáticas (NE 2) e um ECAC (NE 2). Não foram identificados estudos relativos a cãibras associadas ao exercício. Os estudos são consensuais na aparente ausência de eficácia do magnésio na prevenção de cãibras idiopáticas. Quanto às cãibras associadas à gravidez, a evidência é inconsistente. Contudo, o uso de amostras pequenas, períodos de follow-up curtos e a heterogeneidade das metodologias (população, tipo de suplemento, posologia, outcomes) comprometem a extrapolação e reduzem a força das conclusões obtidas.

Conclusão: Perante a evidência encontrada, a suplementação oral de magnésio na população geral parece não ser eficaz na prevenção de cãibras idiopáticas (SORT B), não havendo evidência que suporte a sua prescrição por rotina na prática clínica. Nas cãibras associadas à gravidez a evidência da suplementação oral de magnésio não é clara (SORT B). Para obter conclusões mais robustas são necessários ECAC de melhor qualidade e com metodologias mais homogéneas.

Palavras-chave: Cãibra; Magnésio; Compostos de magnésio.

\section{INTRODUÇÃO}

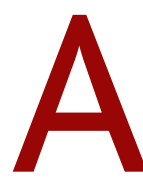
s cãibras musculares são contraturas musculares súbitas, dolorosas, palpáveis e involuntárias que podem durar segundos a minutos, ${ }^{1}$ afetando normalmente os músculos gastrocnémios. ${ }^{2}$ Podem ser classificadas em três entidades: idiopáticas, incluindo cãibras noturnas nas pernas; parafisiológicas, associadas à gravidez ou induzidas pelo

1. Médica Interna de Medicina Geral e Familiar. USF Camélias, ACeS Gaia. 2. Médico Interno de Medicina Geral e Familiar. USF Nova Via, ACeS Espinho/Gaia. exercício; e sintomáticas, relacionadas com fatores etiológicos como medicação ou doenças. ${ }^{3}$

De acordo com estudos europeus, as cãibras atingem 37-60\% da população, ${ }^{4-7}$ tendo uma prevalência ainda superior em indivíduos mais velhos. ${ }^{5}$ Foi descrita uma maior frequência dos sintomas durante a noite (73\%), sendo os músculos mais afetados os da perna (83\%). ${ }^{5}$ Quando frequentes, as cãibras podem causar um sofrimento significativo e perturbação do sono..$^{8-9}$

As cãibras musculares podem ocorrer em vários contextos. São frequentemente observadas em indivíduos 


\begin{tabular}{|c|c|c|}
\hline & Critérios de inclusão & Critérios de exclusão \\
\hline População & Indivíduos adultos com cãibras musculares & $\begin{array}{l}\text { Idade pediátrica } \\
\text { Cãibras musculares relacionadas com fatores } \\
\text { etiológicos como medicação ou doença (e.g., doença } \\
\text { do neurónio motor) }\end{array}$ \\
\hline Intervenção & Suplementação oral de magnésio & Formulação não oral \\
\hline Comparação & Placebo; não tratamento & \\
\hline Outcome & Frequência e intensidade de sintomas & \\
\hline Tipo de estudo & $\begin{array}{l}\text { Meta-análises, revisões sistemáticas, ensaios clínicos } \\
\text { aleatorizados e controlados, estudos de coorte e } \\
\text { caso-controlo e normas de orientação clínica }\end{array}$ & \\
\hline
\end{tabular}

mais velhos e grávidas (em particular no terceiro trimestre), nos quais costumam ocorrer em repouso, ${ }^{1} \mathrm{e}$ também durante ou logo após exercício físico intenso. ${ }^{10}$ Podem ainda estar associadas a doenças do neurónio motor (e.g., esclerose lateral amiotrófica), insuficiência renal ou hepática, hipomagnesemia, hipocalcemia, hipotiroidismo, medicação (e.g., diuréticos, beta-agonistas) e hemodiálise.,11

Uma vez que a deficiência de magnésio foi associada a excitabilidade neuronal e a um incremento da transmissão neuromuscular e a sua reposição se provou eficaz no tratamento de convulsões no contexto de eclâmpsia, foi equacionado um potencial benefício da sua suplementação nas cãibras musculares. ${ }^{12}$ Neste sentido, estão disponíveis vários suplementos de venda livre que consistem em sais de magnésio combinados com citrato, lactato, gluconato, malato, orotato, cloreto, óxido, carbonato, hidróxido, sulfato ou combinações destes aniões, estando disponíveis sob a forma de comprimido, suspensão líquida ou pó. ${ }^{1}$

Contudo, embora estes suplementos de magnésio sejam ativamente comercializados na Europa, ${ }^{8}$ permanecem dúvidas quanto à sua eficácia. Assim sendo, com este estudo pretendemos rever a evidência sobre a eficácia da suplementação oral de magnésio na prevenção de cãibras musculares em indivíduos adultos.

\section{MÉTODOS}

Foi realizada uma pesquisa no dia 4 de julho de 2018 nas bases de dados National Guideline Clearinghouse,
Canadian Medical Association Practice Guidelines Infobase, Guidelines Finder, The Cochrane Library, DARE, Bandolier, Evidence-Based Medicine Online e PubMed com os termos MeSH muscle cramp, magnesiume magnesium compounds, de artigos publicados desde 1 de janeiro de 2008 nas línguas portuguesa, inglesa e espanhola. Os critérios de seleção de artigos utilizados estão descritos no Quadro I.

Para atribuir os níveis de evidência (NE) e forças de recomendação foi utilizada a Strength of Recommendation Taxonomy (SORT), da American Academy of Family Physicians.

\section{RESULTADOS}

Dos 47 artigos encontrados foram excluídos 38 após leitura do título e resumo e quatro após leitura integral do artigo. Deste modo, foram selecionados cinco artigos (uma MA, três RS e um ECAC). O processo de seleção dos estudos incluídos encontra-se descrito na Figura 1 .

A MA de Sebo e colaboradores, ${ }^{12}$ publicada em 2014 (Quadro II), teve como objetivo rever a eficácia do magnésio no tratamento de cãibras noturnas nas pernas e os efeitos secundários desta terapêutica comparativamente ao placebo. Incluiu seis ECAC ( $n=315)$ que compararam a terapêutica com magnésio oral ao placebo. Desses, três incluíram apenas mulheres grávidas. As amostras foram pequenas, sendo que a maior era de 80 participantes. Foram utilizadas diferentes formulações (citrato, lactato, bisglicenato) e doses de magnésio (200 


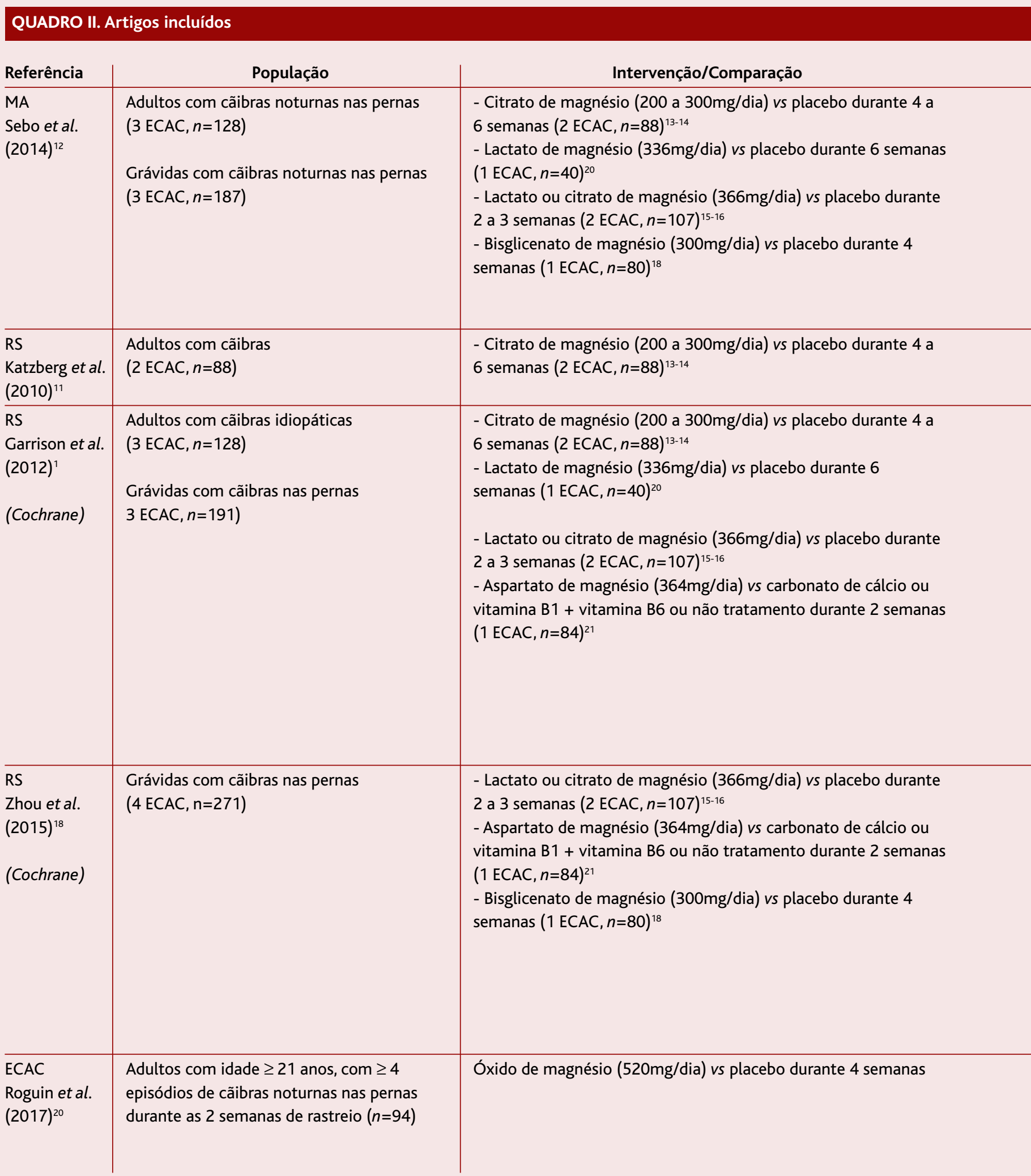


- Apenas dois ensaios, ambos em grávidas, mostraram uma redução estatisticamente significativa na frequência $(p<0,0515$; $p=0,00719)$ e intensidade $\left(p<0,05^{15} ; p=0,04^{19}\right)$ das cãibras a favor do magnésio;

- A nível global, a diferença na mediana do número de cãibras/semana entre o magnésio e o placebo foi de 0,345 (quantil 2,5\%: $-0,133$, quantil 97,5\%: 0,875);

- Os ensaios relativos a uma população mais velha mostraram uma diferença de mediana do número de cãibras por semana de 0,362 (quantil $2,5 \%-0,386$; quantil $97,5 \%$ 1,148) relativamente ao placebo;

- Os três estudos nas grávidas mostraram uma diferença de mediana do número de cãibras por semana de 0,807 (quantil 2,5\% 0,015; quantil 97,5\% 1,207) relativamente ao placebo;

- Não houve diferenças estatisticamente significativas na frequência de efeitos gastrointestinais relativamente ao placebo (MD 0,13; IC95\% -0,51 a 0,78; $p=0,07$ ).

Um dos ensaios concluiu não ter havido melhoria significativa no número de cãibras nos doentes a fazer tratamento com magnésio oral $(p=0,07) ;{ }^{14}$ outro ensaio concluiu que o tratamento não foi superior ao placebo na redução do número $(p=0,59)$ e gravidade $(p=0,84)$ das cãibras. ${ }^{13}$

Nos adultos com cãibras idiopáticas (a maioria das quais eram cãibras noturnas em adultos mais velhos):

- Não houve diferenças estatisticamente significativas entre o magnésio e o placebo na frequência de cãibras, após 4 semanas de tratamento, nos outcomes "percentagem de alteração do número de cãibras por semana relativamente ao número basal" $(-3,93 \%$, IC $95 \%-21,12 \%$ a $13,26 \% ; p=0,65)$ e na "diferença no número de cãibras por semana" $(0,01$ cãibras/semana, IC $95 \%-0,52$ a 0,$55 ; p=0,96)$;

- Não houve diferenças estatisticamente significativas entre o magnésio e o placebo na intensidade (MD -0,04; IC95\% $-0,18$ a $0,11, p=0,62$ ) das cãibras, após 4 semanas de tratamento;

Nas grávidas com cãibras nas pernas:

- O ensaio que comparou o magnésio com não tratamento não encontrou uma redução estatisticamente significativa na frequência ou intensidade das cãibras; ${ }^{22}$

- Dos dois ensaios que compararam o magnésio ao placebo, um não encontrou diferenças significativas entre os dois grupos na frequência ou intensidade das cãibras $\left(p=0,27\right.$ e $p=0,46$, respetivamente) ${ }^{16}$ ao passo que o outro encontrou diferenças significativas em ambos a favor do magnésio ( $p<0,05$ em ambos) $;^{15}$

- Não houve diferenças estatisticamente significativas na suspensão do tratamento por efeitos adversos relativamente ao placebo (-3\%; IC95\% - 10\% a 3\%; $p=0,35)$.

Relativamente à redução da frequência de cãibras:

- Houve diferenças a favor do magnésio no número médio de grávidas a fazer tratamento com magnésio que referiu "não ter cãibras" (RR=5,66; IC95\% 1,35 a 23,68; $p=0,018)$, "ter apenas duas cãibras por semana" (RR=0,29; IC95\% 0,11 a 0,80; $p=0,017)$ e "redução do número de cãibras em 50\%" (RR=1,42; IC95\% 1,09 a 1,86; $p=0,010)$;

- Não houve diferenças entre os dois grupos relativamente ao número total de cãibras durante duas semanas de tratamento (MD 1,80; IC95\% -1,32 a 4,92; $p=0,26)$ nem no número médio de grávidas a fazer tratamento com magnésio que referiu "cãibras diárias" (RR=1,20; IC95\% 0,45 a 3,21; $p=0,71)$, "cãibras dia sim, dia não" (RR=0,44; IC95\% 0,12 a $1,57 ; p=0,21)$ ou "uma cãibra por semana" (RR=1,54; IC95\% 0,62 a 3,87; $p=0,35)$;

- A evidência do magnésio na redução de intensidade das cãibras foi inconclusiva; dois ensaios sugerem haver diferenças a favor do magnésio (RR=1,43; IC95\% 0,99 a 2,06; $p=0,055)^{19}$; MD -17,50; IC95\% -34,68 a -0,32; $\left.p=0,046\right)^{15}$; um ensaio não mostrou diferença em relação ao placebo (MD 1,80; IC95\% -3,10 a 6,70; $p=0,47) ;{ }^{16}$

- Não houve diferenças nos efeitos laterais relativamente ao placebo/não tratamento.

- Houve uma redução estatisticamente significativa $(p<0,001)$ do número médio de cãibras noturnas por semana após o tratamento tanto no grupo do óxido de magnésio $(48,4 \%)$ como no do placebo $(29,5 \%)$;

- Entre os dois grupos não houve diferença significativa no número médio de cãibras por semana $(p=0,67)$ e gravidade $(p=0,38)$ das cãibras noturnas;

- Os efeitos laterais minor, maioritariamente gastrointestinais, ocorreram em ambos os grupos. 


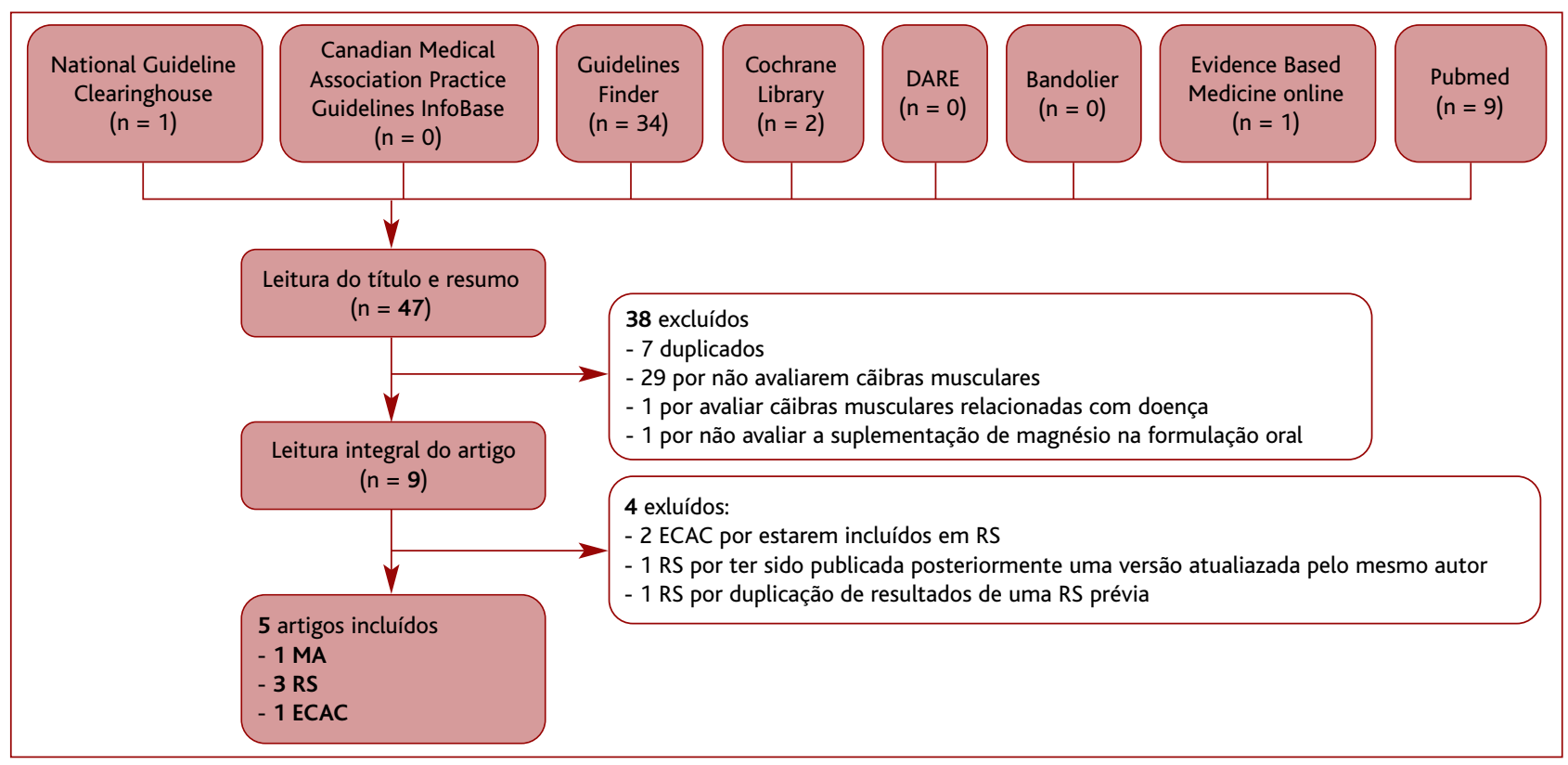

Figura 1. Esquema de seleção dos artigos incluídos.

Legenda: $\mathrm{ECAC}=$ Ensaio clínico aleatorizado e controlado; $\mathrm{MA}=$ Meta-analise; $\mathrm{RS}=$ Revisão sistemática.

a 366mg/dia de magnésio elementar) e a duração do tratamento variou entre cinco dias e seis semanas. Dado que as medidas de outcome variaram consideravelmente entre os estudos não foi possível realizar uma meta-análise padrão para agrupar os seus resultados, pelo que foram realizadas simulações. Os autores concluíram que, na população em geral, a terapêutica com magnésio não parece ser eficaz no tratamento de cãibras noturnas nas pernas, mas que pode haver um pequeno efeito nas grávidas.

A RS de Katzberg e colaboradores, ${ }^{11}$ publicada em 2010 (Quadro II), teve como objetivo rever a evidência da eficácia de diferentes tratamentos sintomáticos de cãibras musculares. Foram excluídas cãibras associadas ao exercício ou à gravidez. Dos 24 artigos incluídos apenas dois ECAC ${ }^{13-14}$ compararam a suplementação de magnésio ao placebo. A ocorrência de efeitos laterais minor foi pouco frequente e semelhante ao grupo controlo e não ocorreram efeitos laterais graves. Os autores concluíram que o magnésio provavelmente não é eficaz no tratamento de cãibras musculares.

A RS de Garrison e colaboradores, ${ }^{1}$ publicada em 2012 (Quadro II), teve como objetivo rever o efeito da suplementação de magnésio comparativamente a não trata- mento, placebo e outros tratamentos em indivíduos com cãibras musculares. Incluiu seis ECAC que compararam a suplementação oral de magnésio versus placebo (cinco ECAC) e versus não tratamento (um ECAC). Três ensaios incluíram apenas cãibras associadas à gravidez ( $n=191)$ e três ensaios somente cãibras idiopáticas ( $n=128)$. Não foram encontrados estudos relativos a cãibras associadas ao exercício físico. Os ensaios usaram diferentes formulações (citrato, lactato e aspartato) e doses de magnésio (200 a 366mg/dia de magnésio elementar). As amostras foram pequenas ( 40 a 84 participantes) e a duração do tratamento variou entre duas e seis semanas. Os dados dos ensaios relativos a cãibras idiopáticas foram estudados através da realização de meta-análise. Contudo, o mesmo não foi possível no caso das cãibras associadas à gravidez dada a heterogeneidade dos respetivos ensaios. Os autores concluíram ser improvável que o magnésio forneça uma profilaxia clinicamente importante de cãibras idiopáticas em adultos mais velhos. Em relação às cãibras associadas à gravidez, a evidência foi controversa, dado que os únicos dois estudos relevantes ${ }^{15-16}$ foram discordantes.

A RS de Zhou e colaboradores, ${ }^{18}$ publicada também em 2015 (Quadro II), teve como objetivo rever a eficá- 
cia e segurança de diferentes intervenções no tratamento de cãibras nas pernas na gravidez. Quatro ECAC compararam a suplementação oral de magnésio durante duas a quatro semanas com placebo (três ECAC) e com não tratamento (um ECAC). Um dos ensaios não especificou a idade gestacional nos critérios de inclusão e nos restantes esta variou entre 14 a 36 semanas. As amostras variaram de 38 a 84 participantes e foram usadas diferentes doses e formulações de magnésio. Dada a inconsistência dos outcomes reportados não foi possível realizar uma meta-análise. A ocorrência de complicações na gravidez, ao nível do parto e neonatais foram pouco reportadas, pelo que não foi possível averiguar a segurança desta intervenção. Os autores concluíram que o magnésio não reduziu de forma consistente a frequência de cãibras nas pernas, tendo mostrado uma redução em dois ensaios ${ }^{15,19} \mathrm{e}$ não mostrando diferença nos outros.

O ECAC duplamente cego de Roguin e colaboradores, ${ }^{20}$ publicado em 2017 (Quadro II), teve como objetivo determinar se o óxido de magnésio era superior ao placebo na profilaxia das cãibras noturnas nas pernas. Foram incluídos indivíduos com mais de 21 anos e com quatro ou mais episódios de cãibras noturnas detetadas na fase de rastreio (duas semanas). Os critérios de exclusão foram gravidez, tratamento atual com quinina, suplementação de magnésio, insuficiência renal e doenças neurológicas major. A amostra foi de 94 participantes, que foram aleatorizados para receber óxido magnésio (520mg de magnésio elementar) ou placebo. Os autores concluíram que o óxido de magnésio não foi superior ao placebo na redução da frequência $(p=0,67)$ ou gravidade $(p=0,38)$ das cãibras noturnas nas pernas, interpretando a redução verificada em ambos os grupos como provável efeito placebo.

\section{CONCLUSÃO}

Perante a evidência encontrada, a suplementação oral de magnésio na população geral parece não ser eficaz na prevenção de cãibras idiopáticas (SORT B), não havendo evidência que suporte a sua prescrição por rotina na prática clínica. Nas cãibras associadas à gravidez, a evidência da suplementação oral de magnésio não é clara (SORT B). No entanto, a robustez destas conclusões está comprometida pela relativa baixa qualidade e heterogeneidade das metodologias. Os critérios de inclusão não foram uniformes, incluindo populações com diferentes faixas etárias e diferentes características de cãibras (qualquer localização ou especificamente nas pernas; qualquer altura do dia ou apenas noturnas). A dimensão reduzida das amostras e a curta duração de tratamento foram limitações transversais a todos os estudos. Quanto ao tipo de intervenção, houve variabilidade entre os estudos na dose de magnésio elementar diária (200-520mg) e no tipo de formulação (citrato, lactato, aspartato, bisglicenato, óxido). Relativamente aos outcomes, embora a maioria dos estudos tenha considerado a diminuição da frequência de cãibras como outcome primário, a forma como essa diminuição foi medida variou (média ou mediana; número absoluto ou percentagem de redução; número de dias e/ou noites com cãibras ou número de cãibras), o que dificulta o agrupamento de dados e a extrapolação de conclusões.

Assim, são necessários ECAC de alta qualidade, com metodologias mais homogéneas, envolvendo um maior número de indivíduos, maior duração de tratamento e de follow-up, para se obter conclusões mais robustas sobre a eficácia e segurança da suplementação de magnésio nas cãibras musculares.

\section{REFERÊNCIAS BIBLIOGRÁFICAS}

1. Garrison SR, Allan GM, Sekhon RK, Musini VM, Khan KM. Magnesium for skeletal muscle cramps. Cochrane Database Syst Rev. 2012;(9): CD009402.

2. Young G. Leg cramps. BMJ Clin Evid. 2009;2009:1113.

3. Parisi L, Pierelli F, Amabile G, Valente G, Calandriello E, Fattapposta F, et al. Muscular cramps: proposals for a new classification. Acta Neurol Scand. 2003;107(3):176-86.

4. Hall AJ. Cramp and salt balance in ordinary life. Lancet. 1947;2(6468): 231-3.

5. Naylor JR, Young JB. A general population survey of rest cramps. Age Ageing. 1994;23(5):418-20.

6. Allen RE, Kirby KA. Nocturnal leg cramps. Am Fam Physician. 2012;86 (4):350-5.

7. Maisonneuve H, Chambe J, Delacour C, Muller J, Rougerie F, Haller DM, et al. Prevalence of cramps in patients over the age of 60 in primary care : a cross sectional study. BMC Fam Pract. 2016;17(1):111.

8. Monderer RS, Wu WP, Thorpy MJ. Nocturnal leg cramps. Curr Neurol Neurosci Rep. 2010;10(1):53-9.

9. Hawke F, Chuter V, Burns J. Impact of nocturnal calf cramping on quality of sleep and health-related quality of life. Qual Life Res. 2013;22(6): 1281-6.

10. Schwellnus MP, Drew N, Collins M. Muscle cramping in athletes: risk factors, clinical assessment, and management. Clin Sports Med. 2008;27(1):183-94 
11. Katzberg HD, Khan AH, So YT. Assessment: symptomatic treatment for muscle cramps (an evidence-based review): report of the Therapeutics and Technology Assessment Subcommittee of the American Academy of Neurology. Neurology. 2010;74(8):691-6.

12. Sebo $P$, Cerutti B, Haller DM. Effect of magnesium therapy on nocturnal leg cramps: a systematic review of randomized controlled trials with meta-analysis using simulations. Fam Pract. 2014;31(1):7-19.

13. Frusso R, Zárate M, Augustovski F, Rubinstein A. Magnesium for the treatment of nocturnal leg cramps: a crossover randomized trial. J Fam Pract. 1999;48(11):868-71.

14. Roffe C, Sills S, Crome P, Jones P. Randomised, cross-over, placebo controlled trial of magnesium citrate in the treatment of chronic persistent leg cramps. Med Sci Monit. 2002;8(5):CR326-30.

15. Dahle LO, Berg G, Hammar M, Hurtig M, Larsson L. The effect of oral magnesium substitution on pregnancy-induced leg cramps. Am J Obstet Gynecol. 1995;173(1):175-80.

16. Nygaard IH, ValbøA, Pethick SV, Bøhmer T. Does oral magnesium substitution relieve pregnancy-induced leg cramps? Eur J Obstet Gynecol Reprod Biol. 2008;141(1):23-6.

17. Young G. Leg cramps. BMJ Clin Evid. 2015;2015:1113.

18. Zhou K, West HM, Zhang J, Xu L, Li W. Interventions for leg cramps in pregnancy. Cochrane Database Syst Rev. 2015;(7):CD010655.

19. Supakatisant C, Phupong V. Oral magnesium for relief in pregnancy- induced leg cramps: a randomised controlled trial. Matern Child Nutr. 2015;11(2):139-45.

20. Maor NR, Alperin M, Shturman E, Khairaldeen H, Friedman M, Karkabi $K$, et al. Effect of magnesium oxide supplementation on nocturnal leg cramps: a randomized clinical trial. JAMA Intern Med. 2017;177(5):61723.

21. Rosenbaum L. Beneficial effects of magnesium supplementation on idiopathic muscle cramps. ClinicalTrials.gov [Internet]; 2012. Available from: https://clinicaltrials.gov/ct2/show/NCT00963638

22. Sohrabvand F, Shariat M, Haghollahi F. Vitamin B supplementation for leg cramps during pregnancy. Int J Gynaecol Obstet. 2006;95(1):48-9.

\section{CONFLITO DE INTERESSES}

Os autores declaram não ter quaisquer conflitos de interesse.

\section{ENDEREÇO PARA CORRESPONDÊNCIA \\ Cátia Palha \\ E-mail: catiapalha@gmail.com \\ https://orcid.org/0000-0002-3194-8756}

Recebido em 23-11-2018

Aceite para publicação em 17-03-2019

\section{ABSTRACT}

\section{ORAL MAGNESIUM SUPPLEMENTATION IN MUSCLE CRAMP PREVENTION: EVIDENCE-BASED REVIEW}

Aim: Evidence review on the efficacy of oral magnesium supplementation in the prevention of muscle cramps without associated pathology, in adults.

Data sources: National Guideline Clearinghouse, Canadian Medical Association Practice Guidelines Infobase, Guidelines Finder, The Cochrane Library, DARE, Bandolier, Evidence-Based Medicine Online, and PubMed.

Methods: On $4^{\text {th }}$ July 2018, using the MeSH terms 'Muscle Cramp', 'Magnesium' and 'Magnesium Compounds', we searched for meta-analyses, systematic reviews, randomized controlled trials (RCT), observational studies and clinical guidelines published in Portuguese, Spanish, and English. To assign levels of evidence (LE) and strength of recommendations, the Strength of Recommendation Taxonomy (SORT) scale of the American Academy of Family Physicians was used.

Results: We found 47 articles, of which five met the inclusion criteria: one meta-analysis (LE 2), three systematic reviews (LE 2) and one RCT (LE 2). No studies related to exercise-associated cramps were found. Studies are consensual in the apparent lack of efficacy of the magnesium in the prevention of idiopathic muscle cramps. As for the pregnancy-associated cramps, the evidence is inconsistent. However, the use of small samples, short follow-up periods and the heterogeneity of methodologies (population, supplement type, posology, and outcomes) compromise the extrapolation and reduce the strength of the conclusions obtained.

Conclusion: According to the evidence found, oral magnesium supplementation in the general population seems to be ineffective in preventing idiopathic muscle cramps (SORT B), therefore, with no evidence to support its routine prescription in clinical practice. In pregnancy-associated cramps, the evidence of oral magnesium supplementation is unclear (SORT B). To achieve more robust conclusions, higher quality RCT is needed, with more homogeneous methodologies.

Keywords: Muscle cramp; Magnesium; Magnesium compounds. 